\title{
Overexpression of klotho in adipose-derived stem cells protects against UVB-induced photoaging in co-cultured human fibroblasts
}

\author{
FURONG FAN ${ }^{1,2}$, YUFEI LI ${ }^{1}$, YI LIU ${ }^{3}$, LI SHAO ${ }^{4}$, JUEHUA YU ${ }^{5}$ and ZIWEI LI $^{4}$ \\ ${ }^{1}$ Department of Plastic and Reconstructive Surgery, The No. 455 Hospital of PLA, Shanghai 200052; \\ ${ }^{2}$ Department of Plastic Surgery, Shanghai East Hospital Affiliated to Tongji University, Shanghai 200120 \\ ${ }^{3}$ Burns and Plastic Surgery Center of CPLA, Lanzhou General Hospital of CPLA, Lanzhou, Gansu 730050; \\ ${ }^{4}$ The VIP Department, East Hospital, Tongji University School of Medicine, Shanghai 200120; \\ ${ }^{5}$ Cancer Research Institute, Zhejiang Cancer Hospital, Hangzhou, Zhejiang 320000, P.R. China
}

Received April 6, 2018; Accepted October 5, 2018

DOI: $10.3892 / \mathrm{mmr} .2018 .9594$

\begin{abstract}
Co-culture methods are widely used in tissue engineering to drive tissue formation with the direct or indirect interaction of multiple cell types. Klotho is a novel biomarker involved in aging. In this study, we evaluated the protective effects of klotho overexpressed adipose-derived stem cells (ADSCs) against ultraviolet radiation B (UVB)-induced photoaging in co-cultured human skin fibroblasts (HSF2 cell line). Furthermore, the involvement of P38 mitogen-activated protein kinase (MAPK) signaling was investigated. ADSCs were isolated from human subcutaneous adipose tissue and the 3rd generation of ADSCs was used after being identified. Klotho overexpression (OE) lentivirus vectors were constructed and identified in ADSCs. The HSF2 cells were seeded in the upper layer of the Transwell co-culture plate $(0.4 \mu \mathrm{m}$ pore polycarbonate membrane) and ADSCs were seeded in the lower layer. UVB irradiation of HSF2 cells was performed using UVB lamps in uncovered petri dishes at room temperature. The present results indicated that the proliferation of ADSCs was increased by klotho OE. Furthermore the proliferation and collagen content of HSF2 were decreased by UVB irradiation in a dose-dependent manner. By contrast, the protein level of matrix metalloproteinases (MMP) 1, 3 and p-P38 in HSF2 were upregulated. In the co-culture system, relative mRNA expression of MMP-1 and MMP-3 as well as protein level of MMP-1, MMP-3 and p-P38 in HSF2 were reduced by co-culture with klotho overexpressed ADSCs when exposed to UVB $\left(20 \mathrm{~mJ} / \mathrm{cm}^{2}\right)$. By contrast, the collagen content of HSF2 was increased. Collectively, OE of klotho in ADSCs notably ameliorates UVB-induced photoaging in co-cultured HSF2,
\end{abstract}

Correspondence to: Dr Furong Fan, Department of Plastic and Reconstructive Surgery, The No. 455 Hospital of PLA, 338 Huaihai West Road, Shanghai 200052, P.R. China

E-mail: fanfr999@126.com

Key words: ADSCs, HSF2, co-culture system, klotho over expression, UVB-induced photoaging, collagen content and these effects were potentially achieved by increasing the collagen content and decreasing the protein level of MMP-1, MMP-3 and p-P38.

\section{Introduction}

Skin aging is caused by various factors such as hormonal imbalance, certain metabolic pathway disorders and, especially, exposure to ultraviolet (UV) irradiation (1). Photoaging mainly results from chronic solar UV irradiation (2), and it is characterized clinically by telangiectasias, dyschromia, roughness, laxity and wrinkle formation (3-5). Ultraviolet radiation B (UVB) $(280-320 \mathrm{~nm})$ is the principal reason for skin photoaging because it can cross the whole epidermis layer and penetrate the dermis (inner layer) of human skin (6), induce keratinocyte apoptosis (7) and reactive oxygen species (ROS)-mediated inflammation (8), and increase the expression and activity of matrix metalloproteinases (MMPs) via mitogen-activated protein kinases (MAPK) signaling pathway $(9,10)$. Overexpression (OE) of MMP-1 (a collagenase) could induce the degradation of extracellular matrix (ECM) by initiating the degradation of type I collagen (COL-I) and type III collagen (COL-III), the major structural protein of ECM, which is secreted by fibroblasts $(11,12)$.

Klotho is a soluble protein and transmembrane that shows glucosidase activity, it serves as a novel biomarker involved in aging (13). Klotho is related to insulin sensitivity, mineralization, cell renewing, reparative processes and electrolytic balance (14). Klotho mutant mice develop a phenotype characterized by accelerated skin atrophy, aging, osteoporosis, lung emphysema, vascular calcification and delayed wound healing (13).

Co-culture methods are widely used in tissue engineering to drive tissue formation with the direct or indirect interaction of multiple cell types (15). Co-culture can effectively recapitulate the relationships among cell types within processes and native tissue that are inefficient when relying solely on soluble factors and scaffolds. On the one hand, when acting as the target cells, stem cells differentiate and eventually synthesize the ECM or metabolites that confer function to a tissue in co-culture systems (15). On the other hand, as 
assisting cells, stem cells can also promote homeostasis of engineered tissues. For instance, stem cells promote the tissue repair directly (16), and they have the ability to inhibit cell apoptosis, locally suppress the immune system and promote cell differentiation and proliferation indirectly $(17,18)$. Thus, in this study, we evaluated the protective effects of klotho overexpressed adipose-derived stem cells (ADSCs) on UVB-induced photoaging in co-cultured human fibroblasts cell line human skin fibroblasts (HSF2) in vitro, and relative phosphorylated P38 MAPK (p-P38) signaling were investigated during this process.

\section{Materials and methods}

Chemicals and reagents. FITC mouse anti-human CD90, CD44, CD45 and CD11b antibodies were purchased from Becton-Dickinson (BD Biosciences, San Jose, CA, USA). Dulbecco's modified Eagle's medium (DMEM) was purchased from Hyclone (GE Healthcare, Logan, UT, USA). Fetal bovine serum (FBS) was obtained from Gibco (Thermo Fisher Scientific, Inc., Waltham, MA, USA). TRIzol reagents were purchased from Invitrogen (Thermo Fisher Scientific, Inc.). ELISA kits of human COL-I, COL-III, MMP1 and MMP3 were purchased from Cell Signaling Technology Inc. (Danvers, MA, USA). Primary antibodies of klotho (1:800; polyclonal, cat. no. ab203576), COL-I (1:2,000; polyclonal, cat. no. ab34710), COL-III (1:5,000; polyclonal, cat. no. ab7778), MMP1 (1:2,000; polyclonal, cat. no. ab38929), MMP3 (1:500; polyclonal, cat. no. ab53015), P38 (1:1,000; polyclonal, cat. no. ab27986) and p-P38 (1:2,000; polyclonal, cat. no. ab47363) were purchased from Abcam (Cambridge, MA, USA). Primary antibody of GAPDH (1:1,000; monoclonal, cat. no. 5174) was purchased from Cell Signaling Technology, Inc. Horseradish peroxidase (HRP)-conjugated goat antirabbit IgG (1:1,000; polyclonal, cat. no. A0208) was purchased from Beyotime Institute of Biotechnology (Shanghai, China).

Cells. HSF2 (cat. no. bio-51608; Beijing Baiou Bowei Biotechnology Co., Ltd., Beijing, China) and 293 (cat. no. bio-72947; Beijing Baiou Bowei Biotechnology Co., Ltd.). Eight human subcutaneous adipose tissue samples of healthy normal weight males (aged, 20-28 years) were collected during physical examination after informed consent was completed and signed by the donors at Shanghai East Hospital Affiliated to Tongji University (Shanghai, China) in June, 2017. One of the samples was used in the present study. ADSCs were isolated from human subcutaneous adipose tissue as previously described (19). This study was approved by the Ethics Committee of Shanghai East Hospital Affiliated to Tongji University. Researchers were blinded to the experimental groups.

The 3rd generation of ADSCs $\left(1 \times 10^{4}\right)$ was incubated for $10 \mathrm{~h}$ at $4^{\circ} \mathrm{C}$ with FITC mouse anti-human CD90, CD44, CD 45 and $\mathrm{CD} 11 \mathrm{~b}$ antibodies, respectively. Secondary antibodies labeled with FITC were added and incubated for $1 \mathrm{~h}$ at $4^{\circ} \mathrm{C}$. The expression of stromal markers (CD11b-FITC, CD44-FITC, CD45-FITC, CD90-FITC) on the cellular membrane was analyzed by flow cytometry (cat. no. E670006-0300, Shanghai, China). The multipotentiality of ADSCs (adipogenic, chondrogenic and osteogenic) were tested. The 3rd generation of ADSCs (approximately $80 \%$ covered) was seeded in a 6 -well culture plate with the adipogenic, chondrogenic and osteogenic differentiation medium (Cyagen Biosciences Inc. Guangzhou, China) for 2 weeks (at $37^{\circ} \mathrm{C}$ with $5 \% \mathrm{CO}_{2}$ ), respectively, according to the manufacturer's protocol. Finally, these ADSCs were stained by Oil Red O, Safranin O and alkaline phosphatase staining kits according to the protocol, and then detected and distinguished by optical microscope (Olympus, Corporation; Tokyo, Japan).

Co-culture system. In the present study, HSF2 cells and ADSCs were co-cultured indirectly in a Transwell co-culture plate $\left(0.4 \mu \mathrm{m}\right.$ polyester film). The HSF2 cells $\left(1 \times 10^{5}\right)$ were seeded in the upper layer and ADSCs $\left(1 \times 10^{5}\right)$ were seeded in the lower layer. The cells were cultured at $37^{\circ} \mathrm{C}$ (DMEM with $10 \% \mathrm{FBS}$ and $5 \% \mathrm{CO}_{2}$ ). Three biological replicates were prepared for each sample and the experiment was repeated 3 times.

UVB irradiation. The HSF2 cells were collected and washed three times with sterile PBS and evenly distributed in PBS in uncovered petri dishes. UVB irradiation was performed using the UVB lamps (LEITUO illumination, Shenzhen, China) in a biosafety cabinet at room temperature $\left(25^{\circ} \mathrm{C}\right)$ and the UV radiation intensity was validated by the UV photometric detector (Deshengxing Technology Co., Ltd., Shenzhen, China). The irradiating distance was set at $15 \mathrm{~cm}$. The UVB irradiation doses were set at 0 (dark treatment), 10, 20 and $40 \mathrm{~mJ} / \mathrm{cm}^{2}$ for different groups respectively. After each UVB irradiation, the cells were washed by PBS then cultured at $37^{\circ} \mathrm{C}$ (DMEM with $10 \% \mathrm{FBS}$ and $5 \% \mathrm{CO}_{2}$ ). The irradiation lasted $60 \mathrm{~min} /$ day for 3 days. Cells in each group were harvested at 0, 24, 48 and $72 \mathrm{~h}$ in radiation experiment.

Construction and identification of lentivirus vectors. The klotho (Homo sapiens klotho; GenBank; AB005142.1) OE/empty plasmid lentivirus vectors were constructed by JRDun Biotech (Shanghai, China). Recombinant lentivirus was generated using AdEasy technology as previously described with minor modifications (20). Recombinant lentivirus was produced and amplified in packaging 293 cells.

Viral supernatants were diluted in culture medium to give the desired concentration and added to logarithmic phase monolayer ADSCs. After being cultured for $48 \mathrm{~h}$, the function of vectors in ADSCs was identified. Relative mRNA expression and protein level of klotho in ADSCs were tested by quantitative PCR, and western blot analysis, respectively.

Effect of klotho OE on the proliferation of ADSCs. ADSCs were divided into 3 groups: ADSCs group (NC), ADSCs + empty plasmid control group (EPC) and ADSCs + klotho OE group (OE). ADSCs in EPC and OE were transduced with klotho $\mathrm{OE}$ and empty plasmid lentivirus respectively. Cells in each group were harvested at $0,24,48$ and $72 \mathrm{~h}$ after incubation. The proliferation of ADSCs at each time-point was tested by CCK-8 assays kit according to a previous report (21).

Effect of UVB irradiation on HSF2. UVB irradiation was carried out using a UV lighter (LEITUO illumination, Shenzhen, China). Immediately after the irradiation, the PBS was aspirated and replaced with complete medium. 

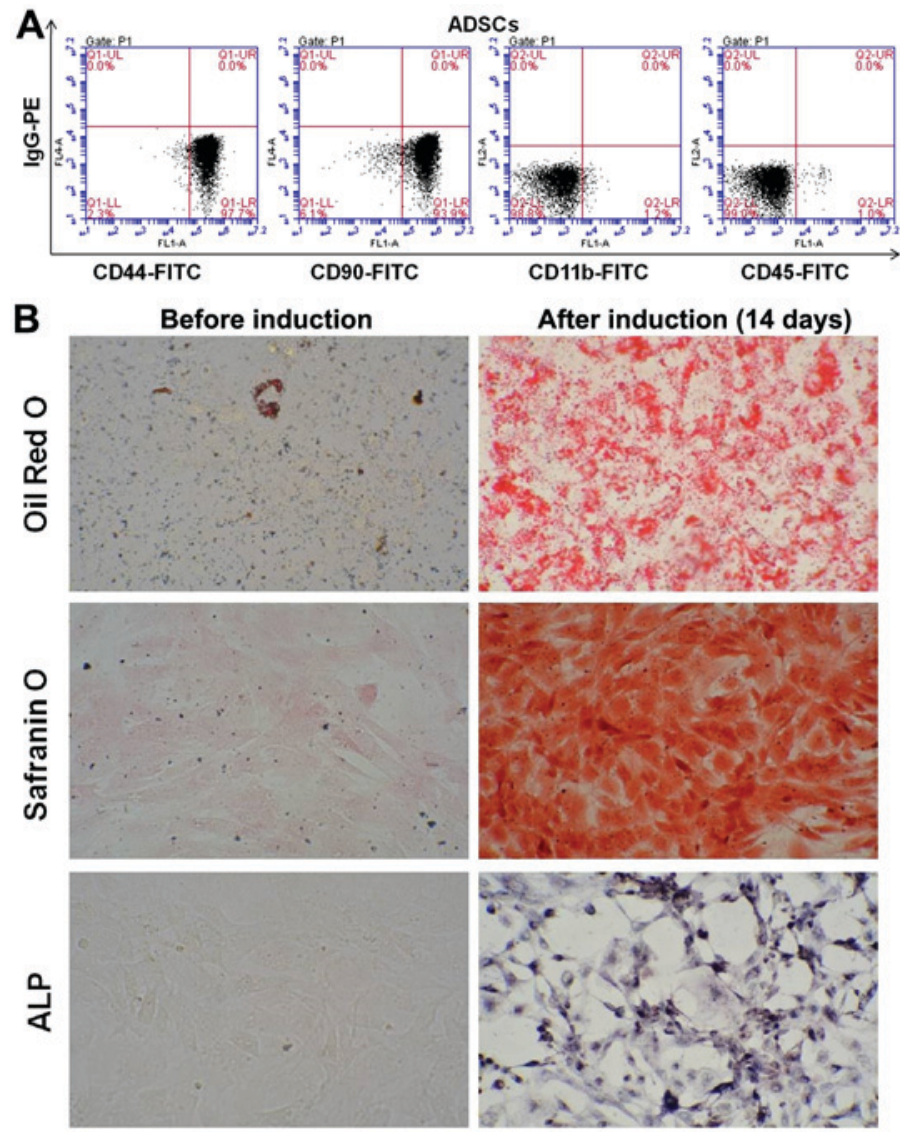

Figure 1. The identification of the 3rd generation ADSCs using flow cytometry. (A) ADSCs were positive for the cell surface markers CD44 (97.7\%) and CD90 (93.9\%) but negative for CD11b (1.1\%) and CD45 (1.0\%) (n=3). (B) The multipotentiality of ADSCs cells. Oil Red O staining of the ADSCs after 2 weeks of culture demonstrated numerous intracellular lipid droplets. Safranin O and alkaline phosphatase staining was positive. Magnification, x200. ADSCs, adipose-derived stem cells.

UVB irradiation doses were set at 0 (dark treatment), 10, 20 and $40 \mathrm{~mJ} / \mathrm{cm}^{2}$ for further experiment. The irradiation lasted $60 \mathrm{~min} /$ day for 3 days. Cells in each group were harvested at 0 , 24,48 and $72 \mathrm{~h}$ in the radiation experiment. The proliferation of HSF2 at each time-point was tested by CCK-8 assays kit according to a previous report (21). The relative expression of MMP1, MMP3, COL-I and COL-III were measured by ELISA kits according to the protocol, and the protein level of P38 and p-P38 was tested by western blot analysis at the end of the experiment.

Effect of klotho overexpressing ADSCs on co-cultured HSF 2 under UVB irradiation. ADSCs were divided into 3 groups: ADSCs, ADSCs + empty plasmid control group and ADSCs + klotho OE group. In ADSCs + empty plasmid control group and ADSCs + klotho OE group, ADSCs were transduced with klotho $\mathrm{OE}$ and empty plasmid lentivirus, respectively. Cells in each group were incubated at normal conditions for $48 \mathrm{~h}$, and these cells were used in the co-culture system.

The co-culture cells were divided into 3 groups: HSF2 + ADSCs (NC), HSF2 + ADSCs + empty plasmid control group (EPC) and HSF2 + ADSCs + klotho OE group (OE). The co-culture system was achieved according to a previous report (22). Briefly, ADSCs and HSF2 were mixed and incubated with complete DMEM containing $10 \%$ FBS with $100 \mathrm{U} / \mathrm{ml}$ penicillin/streptomycin in a humidified
Table I. Primers used in qPCR assays.

\begin{tabular}{lll}
\hline Genes & \multicolumn{1}{c}{ Sequence (5'-3') } & Description \\
\hline MMP1 & ATTCTACTGATATCGGGGCTTTGA & $\mathrm{F}$ \\
MMP1 & ATGTCCTTGGGGTATCCGTGTAG & $\mathrm{R}$ \\
MMP3 & TATGGATCCCCCCTGACTCCCCTGAG & $\mathrm{F}$ \\
MMP3 & ATGGAATTCAGGTTCAAGCTTCCTGAGG & $\mathrm{R}$ \\
Klotho & CACGGCAAGGGTGCGTCCAT & $\mathrm{F}$ \\
Klotho & TCGCGCCCACGAGATGGAGA & $\mathrm{R}$ \\
GAPDH & CTCATGACCACAGTCCATGC & $\mathrm{F}$ \\
GAPDH TTCAGCTCTGGGATGACCTT & $\mathrm{R}$ \\
\hline
\end{tabular}

F, forward; R, reverse.

atmosphere at $37^{\circ} \mathrm{C}$ with $5 \% \mathrm{CO}_{2}$. UVB irradiation dose was set at $20 \mathrm{~mJ} / \mathrm{cm}^{2}$. The irradiation lasted $60 \mathrm{~min} /$ day for 3 days. HSF2 cells were separated and collected at the end of experiment. Relative mRNA expression of MMP1 and MMP3 were measured by quantitative PCR. Protein level of MMP1, MMP3, COL- I, COL-III, P38 and p-P38 were tested by western blot analysis.

$R T-q P C R$. The level of the mRNA expression was determined using qPCR. The total RNA ( $2 \mu \mathrm{g}$ of each sample) was extracted 


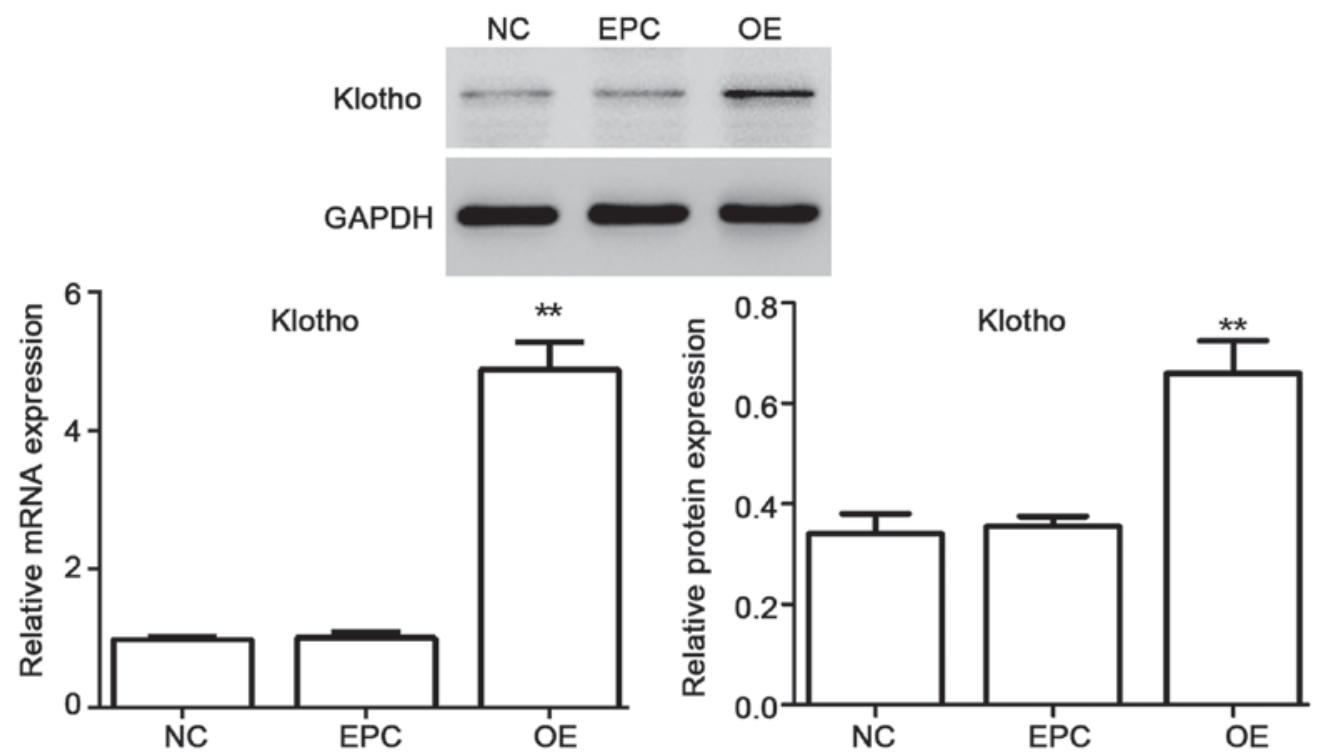

Figure 2. The identification of klotho OE lentivirus vectors. The mRNA expression as well as protein level of klotho was significantly upregulated by klotho OE in ADSCs. NC, ADSCs group; EPC, ADSCs + empty plasmid control group; OE, ADSCs + klotho OE group. ${ }^{*}<0.05,{ }^{* *} \mathrm{P}<0.01 \mathrm{compared}$ with EPC (n=3). $\mathrm{OE}$, overexpression; ADSCs, adipose-derived stem cells; EPC, empty plasmid control group.

and reverse transcribed to cDNA by the reverse transcription kits (Thermo Fisher Scientific, Inc.). The obtained cDNA was used as template for RT-qPCR analysis on quantitative real-time PCR machine (ABI-7300) with SYBR-Green reagents (Thermo Fisher Scientific, Inc.).

Primers (Table I) for the qPCR were designed by Primer 5.0 and synthesized by JRDun Biotech. Relative mRNA expression was evaluated by $2^{-\Delta \Delta C q}$ relative quantitative analysis in each sample against GAPDH gene expression. $\Delta \Delta \mathrm{Cq}=(\mathrm{Cq}$, target gene in the treated group - Cq, reference gene in the treated group) - (Cq, target gene in the control group - Cq, reference gene in the control group) (23).

Western blot analysis. The protein levels of MMP1, MMP3, COL-I, COL-III, P38 and p-P38 were tested by western blot analysis. Total protein was extracted with Cell Protein Extraction Reagent (cat. no. 89802, Thermo Fisher Scientific, Inc.). Total protein was determined using BCA Protein Assay kit (cat. no. 23227, Thermo Fisher Scientific, Inc.). Protein $(35 \mu \mathrm{g})$ was added per lane and separated by $10 \%$ SDS-PAGE and then transferred onto PVDF membranes as previously described (24). The membranes were blocked with 5\% fat-free dry milk at $25^{\circ} \mathrm{C}$ for $1 \mathrm{~h}$ then incubated with the respective primary antibody (MMP1, MMP3, COL-I, COL-III, P38, p-P38 and GAPDH) overnight at $4^{\circ} \mathrm{C}$, followed by a secondary antibody [horseradish peroxidase (HRP)-conjugated goat anti-rabbit $\mathrm{IgG}]$ for $2 \mathrm{~h}$ at $4^{\circ} \mathrm{C}$. Finally, the protein bands were detected by an ECL-detecting kit (Beyotime Institute of Biotechnology). Each blot was normalized to its corresponding internal control-GAPDH value. Band intensities were quantifed by densitometry using Image-Pro plus Software version 6.0 (Media Cybernetics, Inc., Rockville, MD, USA).

Statistical analysis. The data are expressed as mean \pm standard deviation. Statistical analyses of data were performed by one-way analysis of variance (ANOVA) and Tukey's honest

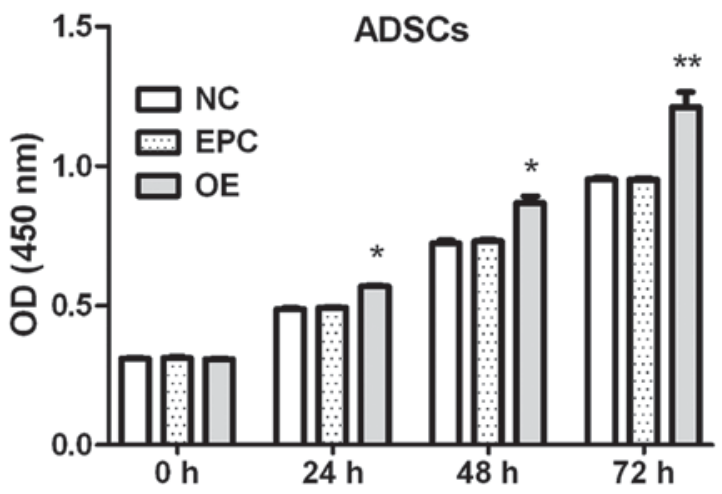

Figure 3. Klotho OE promotes the proliferation of ADSCs. NC, ADSCs group; EPC, ADSCs + empty plasmid control group; OE, ADSCs + klotho OE group. ${ }^{*} \mathrm{P}<0.05,{ }^{* *} \mathrm{P}<0.01$ compared with EPC $(\mathrm{n}=3)$. OE, overexpression; ADSCs, adipose-derived stem cells; EPC, empty plasmid control group.

significant difference (HSD) post hoc test. Data analysis was carried out using SPSS 20.0 software (IBM Corp., Armonk, NY, USA). $\mathrm{P}<0.05$ was considered to indicate a statistically significant difference.

\section{Results}

Identification of ADSCs. The flow cytometry results indicated that CD44- and CD90-positive cells were $97.7 \%$ and $93.9 \%$, while CD11b (1.1\%) and CD45 (1.0\%) were negative on the 3rd generation ADSCs (Fig. 1A). The results suggested that the majority of these cells are ADSCs after three generation subculture. Oil Red O staining of the ADSCs after 2 weeks of culture demonstrated numerous intracellular lipid droplets. Safranin $\mathrm{O}$ and alkaline phosphatase staining was positive (Fig. 1B). Thus, our experiment successfully isolated ADSCs, the cell surface marks were consistent with the characteristics of ADSCs. The study successfully induced ADSCs to adipogenic, chondrogenic and osteogenic cells. The isolated 

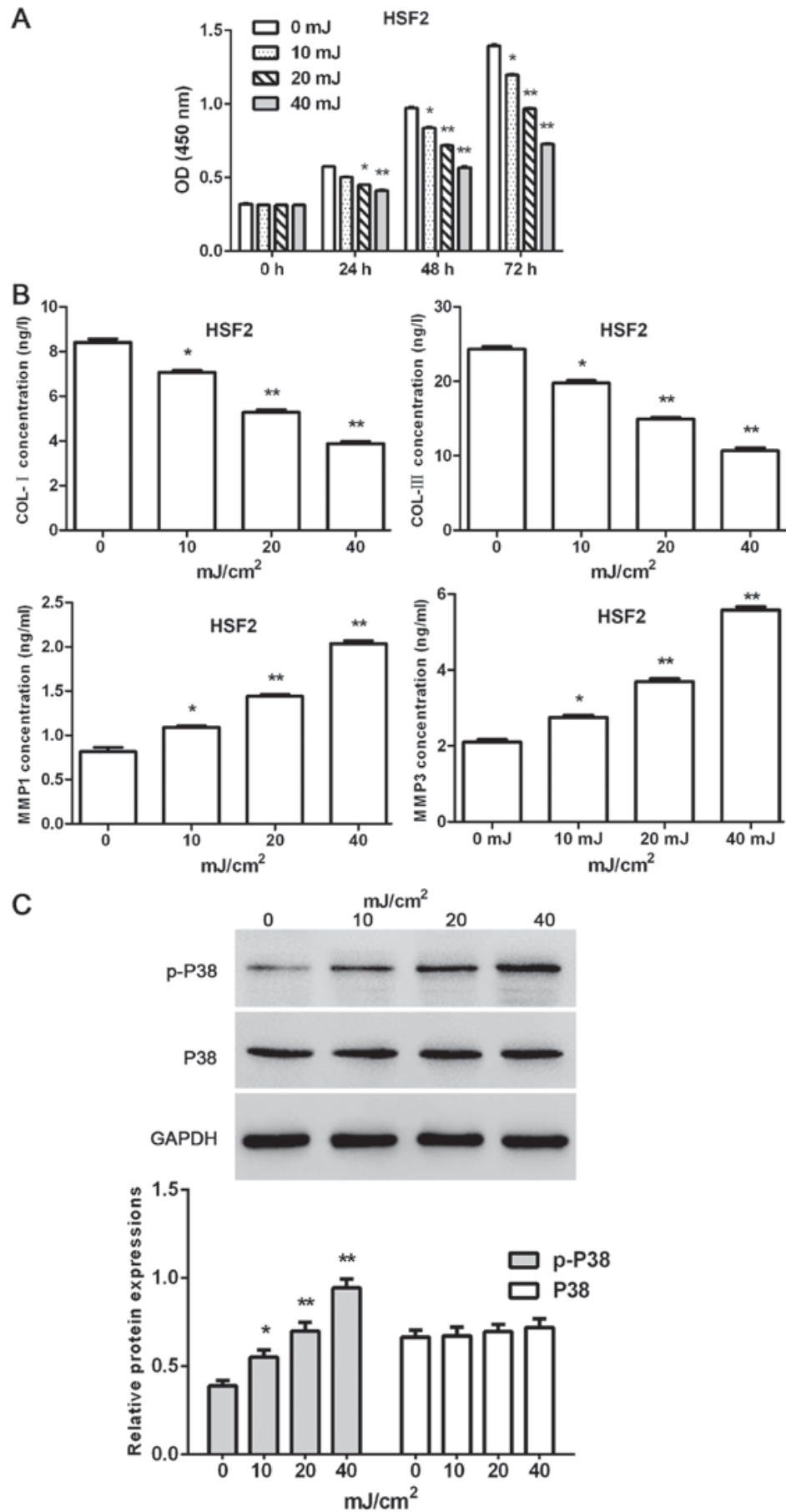

Figure 4. Effect of UVB irradiation on HSF2. (A) UVB irradiation reduces the proliferation of HSF2 cells in a time- and dose-dependent manner. (B) UVB irradiation decreased the concentrations of COL-I and COL-III, and increased the concentrations of MMP1 and MMP3 in HSF2 cells. (C) UVB irradiation dose-dependently upregulated the protein level of $\mathrm{p}-\mathrm{P} 38 .{ }^{*} \mathrm{P}<0.05,{ }^{* *} \mathrm{P}<0.01$ compared with $0 \mathrm{~mJ} / \mathrm{cm}^{2}(\mathrm{n}=3)$. UVB, ultraviolet radiation $\mathrm{B}$; HSF2, human skin fibroblasts.

ADSCs have the ability of multi-potential differentiation and it could be used in the co-culture system.

Identification of lentivirus vectors. Viral supernatants were diluted in culture medium to give the desired concentration and added to logarithmic phase monolayer cell cultures for $48 \mathrm{~h}$. Then the function of vectors in ADSC cells was identified. The mRNA expression as well as protein level of klotho was tested. As shown in Fig. 2, the mRNA expression as well as protein level of klotho was significantly upregulated for klotho OE in ADSCs.
Klotho OE promotes the proliferation of ADSCs. Effects of klotho OE on the proliferation of ADSCs at each time-point were measured by CCK- 8 assay.

The proliferation of ADSCs was increased by klotho OE (8.2\% at $24 \mathrm{~h}, 14.5 \%$ at $48 \mathrm{~h}$ and $25.8 \%$ at $72 \mathrm{~h}$ compared to EPC group) (Fig. 3).

Effect of UVB irradiation on HSF2. The proliferation of HSF2 at $0,24,48$ and $72 \mathrm{~h}$ was tested by CCK- 8 assays kit after various doses of UVB irradiation. Moreover, concentration of 

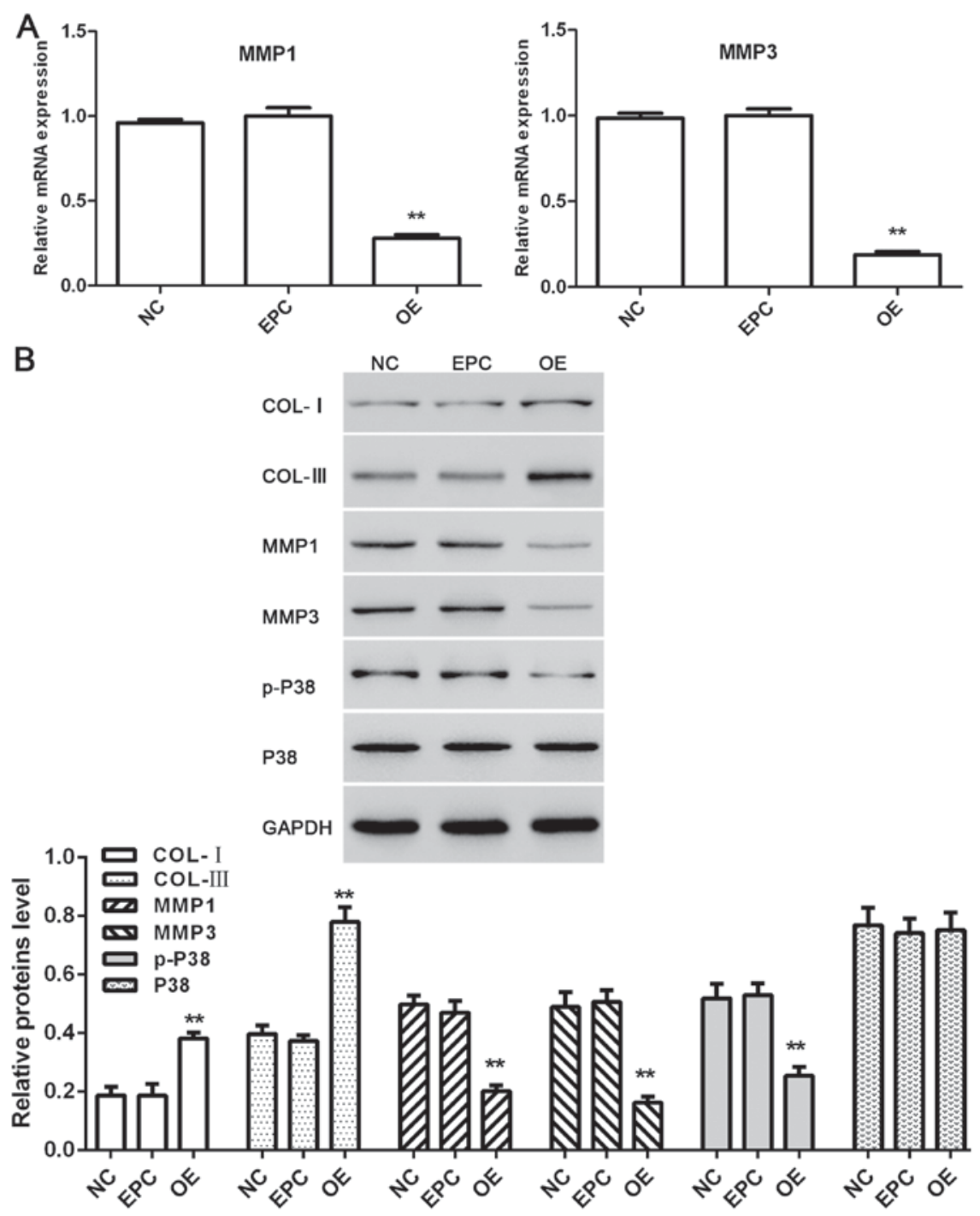

Figure 5. Effect of klotho overexpressed ADSCs on co-cultured HSF2 under UVB irradiation. (A) Relative mRNA expressions of MMP1 and MMP3 in HSF2 cells. (B) Proteins level of COL-I, COL-III, MMP1, MMP3, p-P38 and P38 in HSF2 cells. NC, HSF2 cells separated from HSF2 + ADSCs group; EPC, HSF2 cells separated from HSF2 + ADSCs + empty plasmid control group; OE, HSF2 cells separated from HSF2 + ADSCs + klotho OE group. " $\mathrm{P}<0.05$, ${ }^{* *} \mathrm{P}<0.01$ compared with EPC $(n=3)$. OE, overexpression; ADSCs, adipose-derived stem cells; HSF2, human skin fibroblasts; EPC, empty plasmid control group.

MMP1, MMP3, COL-I and COL-III were measured by ELISA kits according to the protocol, and the protein level of P38 and p-P38 were tested by western blot analysis at the end of the experiment.

The present results indicated that the proliferation of HSF 2 cells was time- and dose-dependently decreased by UVB irradiation (except UVB $10 \mathrm{~mJ} / \mathrm{cm}^{2}$ at the time-point of $24 \mathrm{~h} ; 7.3,13.5$ and $19.9 \%$ for UVB $10 \mathrm{~mJ} / \mathrm{cm}^{2}$ at 24,48 and $72 \mathrm{~h} ; 12.6,25.5$ and $42.8 \%$ for UVB $20 \mathrm{~mJ} / \mathrm{cm}^{2} 24,48$ and $72 \mathrm{~h} ; 16.3,40.5$ and $66.7 \%$ for UVB $10 \mathrm{~mJ} / \mathrm{cm}^{2} 24,48$ and 72 h, respectively) (Fig. 4A). Furthermore, UVB irradiation dose-dependently reduced the concentrations of COL-I and COL-III, and increase the concentrations of MMP1 and MMP3 in HSF2 cells (Fig. 4B). Furthermore, the protein level of p-P38 in HSF2 cells was dose-dependently upregulated by UVB irradiation. By contrast, the protein level of P38 showed no changes when exposed to UVB irradiation (Fig. 4C).

Effect of klotho overexpressed ADSCs on co-cultured HSF2 under UVB irradiation. HSF2 were separated from the co-culture system at the end of experiment. Relative mRNA expression of MMP1 and MMP3 as well as protein level of COL-I, COL-III, MMP1, MMP3, p-P38 and P38 in each group were tested.

As shown in Fig. 5A, the mRNA expression of MMP1 and MMP3 as well as the protein level of MMP1, MMP3 and p-P38 in HSF2 cells were downregulated by klotho-overexpressed ADSCs in the co-culture system exposed to UVB irradiation. By contrast, the protein level of COL-I and COL-III were upregulated, and the protein level of P38 had no obvious change (Fig. 5B).

\section{Discussion}

UV rays from the sun are a common environmental factor affecting humans. Chronic UV irradiation could lead to skin cancer $(25,26)$, accelerated aging of the skin $(27,28)$, cataract $(29,30)$ and immunosuppression $(31,32)$. Photoaging skin is characterized clinically by coarseness, laxity and wrinkles, and this is closely associated with disorganization of collagen 
fibers and reduction in collagen content $(33,34)$. On the other hand, UVB irradiation-induced ROS mediate the phosphorylation of protein kinases through MAPK signaling pathway (9). ROS can cause upregulation of the expression and activity of MMPs, which is associated with collagen degradation in photoaged skin $(35,36)$. The present results show that the proliferation and the collagen content of HSF2 were decreased by UVB irradiation. By contrast, the protein level of MMP1, MMP3 and p-P38 in HSF2 were upregulated.

Klotho is an aging suppressor gene, and its $\mathrm{OE}$ in mice extends the life span of the animals in vivo (37). Furthermore, klotho regulates cellular stress responses to oxidative stress by inhibiting insulin/IGF-1 signaling pathway (38), enhancing FoxO forkhead transcription factor FoxO3-mediated manganese superoxide dismutase expression by negatively regulating PI3K/AKT pathway (39) and suppressing Nox 2 by cAMP/PKA pathway (40). In addition, klotho expression was inhibited by activating $\mathrm{NF}-\kappa \mathrm{B}$ signaling in melanoma cells (41). Stem cells are the future in tissue regeneration and engineering. In the cell co-culture system, stem cells provide a target cell source with multipotent differentiation capacity, as well as assisting cells that promote tissue metabolism, homeostasis, repair and growth. Their incorporation into co-culture systems seems to play an important role in the formation of complex tissues or organs (15). In the present study, ADSC cells act as assisting cells, and the protective effect was achieved indirectly. The positive effects come from the soluble klotho and various growth factors secreted by klotho overexpressed ADSC cells.

In conclusion, $\mathrm{OE}$ of klotho in ADSCs ameliorate UVB-induced photoaging in co-cultured HSF2 in vitro, and these antiaging effects were potentially achieved by increasing the collagen content and decreasing the protein level of MMP-1, MMP-3 and p-P38. Future longitudinal research is required to explore the function of ADSCs in skin repair and functional reconstruction in vivo.

\section{Acknowledgements}

Not applicable.

\section{Funding}

No funding was received.

\section{Availability of data and materials}

The datasets used and/or analyzed during the present study are available from the corresponding author on reasonable request.

\section{Authors' contributions}

FF was involved in drafting the manuscript and cell culture. YuL helped with UVB irradiation. YiL constructed the vector. LS and JY were responsible for CCK-8 assays. ZL contributed to ELISA. All authors read and approved the final study.

\section{Ethics approval and consent to participate}

The study was approved by the Ethics Committee of Shanghai East Hospital Affiliated to Tongji University (Shanghai,
China). Signed informed consents were obtained from the patients.

\section{Patient consent for publication}

Not applicable.

\section{Competing interests}

The authors declared that they have no conflicts of interest in this study.

\section{References}

1. Huang CY, Lin YT, Kuo HC, Chiou WF and Lee MH: Compounds isolated from Eriobotrya deflexa leaves protect against ultraviolet radiation B-induced photoaging in human fibroblasts. J Photochem Photobiol B 175: 244-253, 2017.

2. Grether-Beck S, Wlaschek M, Krutmann J and Scharffetter -Kochanek K: Photodamage and photoaging - prevention and treatment. J Dtsch Dermatol Ges 3 (s2): S19-S25, 2005 (In German).

3. Rokhsar CK, Lee S and Fitzpatrick RE: Review of photorejuvenation: Devices, cosmeceuticals, or both? Dermatol Surg 31: 1166-1178, discussion 1178, 2005.

4. Goldman MP, Weiss RA and Weiss MA: Intense pulsed light as a nonablative approach to photoaging. Dermatol Surg 31: 1179-1187, discussion 1187, 2005.

5. Munavalli GS, Weiss RA and Halder RM: Photoaging and nonablative photorejuvenation in ethnic skin. Dermatol Surg 31: 1250-1260, discussion 1261, 2005.

6. Nichols JA and Katiyar SK: Skin photoprotection by natural polyphenols: Anti-inflammatory, antioxidant and DNA repair mechanisms. Arch Dermatol Res 302: 71-83, 2010.

7. Lei X, Liu B, Han W, Ming M and He YY: UVB-Induced p21 degradation promotes apoptosis of human keratinocytes. Photochem Photobiol Sci 9: 1640-1648, 2010.

8. Duncan FJ, Martin JR, Wulff BC, Stoner GD, Tober KL, Oberyszyn TM, Kusewitt DF and Van Buskirk AM: Topical treatment with black raspberry extract reduces cutaneous UVB-induced carcinogenesis and inflammation. Cancer Prev Res (Phila) 2: 665-672, 2009.

9. Sharma SD, Meeran SM and Katiyar SK: Dietary grape seed proanthocyanidins inhibit UVB-induced oxidative stress and activation of mitogen-activated protein kinases and nuclear factor-kappaB signaling in in vivo SKH-1 hairless mice. Mol Cancer Ther 6: 995-1005, 2007.

10. Jin XJ, Kim EJ, Oh IK, Kim YK, Park CH and Chung JH: Prevention of UV-induced skin damages by 11,14,17-eicosatrienoic acid in hairless mice in vivo. J Korean Med Sci 25: 930-937, 2010.

11. Lee YL, Lee MH, Chang HJ, Huang PY, Huang IJ, Cheng KT and Leu SJ: Taiwanese native plants inhibit matrix metalloproteinase-9 activity after ultraviolet B irradiation. Molecules 14: 1062-1071, 2009.

12. Kähäri VM and Saarialho-Kere U: Matrix metalloproteinases in skin. Exp Dermatol 6: 199-213, 1997.

13. Lanske B and Razzaque MS: Premature aging in klotho mutant mice: Cause or consequence? Ageing Res Rev 6: 73-79, 2007

14. Talotta R, Bongiovanni S, Letizia T, Rigamonti F, Ditto MC, Atzeni F, Salaffi F, Batticciotto A, Gerardi MC, Antivalle M, et al: Measurement of serum klotho in systemic sclerosis. Dis Markers 2017: 9545930, 2017.

15. Paschos NK, Brown WE, Eswaramoorthy R, Hu JC and Athanasiou KA: Advances in tissue engineering through stem cell-based co-culture. J Tissue Eng Regen Med 9: 488-503, 2015.

16. Orlic D, Kajstura J, Chimenti S, Limana F, Jakoniuk I, Quaini F, Nadal-Ginard B, Bodine DM, Leri A and Anversa P: Mobilized bone marrow cells repair the infarcted heart, improving function and survival. Proc Natl Acad Sci USA 98: 10344-10349, 2001.

17. Caplan AI and Dennis JE: Mesenchymal stem cells as trophic mediators. J Cell Biochem 98: 1076-1084, 2006.

18. Scadden DT: The stem-cell niche as an entity of action. Nature 441: 1075-1079, 2006. 
19. Zuk PA, Zhu M, Mizuno H, Huang J, Futrell JW, Katz AJ, Benhaim P, Lorenz HP and Hedrick MH: Multilineage cells from human adipose tissue: Implications for cell-based therapies. Tissue Eng 7: 211-228, 2001.

20. Luo J, Tang M, Huang J, He BC, Gao JL, Chen L, Zuo GW, Zhang W, Luo Q, Shi Q, et al: TGFbeta/BMP type I receptors ALK1 and ALK2 are essential for BMP9-induced osteogenic signaling in mesenchymal stem cells. J Biol Chem 285 29588-29598, 2010.

21. Shen T, Shen J, Zheng QQ, Li QS, Zhao HL, Cui L and Hong CY: Cell viability and extracellular matrix synthesis in a co-culture system of corneal stromal cells and adipose-derived mesenchymal stem cells. Int J Ophthalmol 10: 670-678, 2017.

22. Xu X, Wang HY, Zhang Y, Liu Y, Li YQ, Tao K, Wu CT, Jin JD and Liu XY: Adipose-derived stem cells cooperate with fractional carbon dioxide laser in antagonizing photoaging: A potential role of Wnt and $\beta$-catenin signaling. Cell Biosci 4: 24, 2014.

23. Livak KJ and Schmittgen TD: Analysis of relative gene expression data using real time quantitative PCR and the 2(-DeltaDeltaC(T)) method. Methods 25: 402 408, 2001.

24. Blumer MJ, Longato S and Fritsch H: Structure, formation and role of cartilage canals in the developing bone. Ann Anat 190: 305-315, 2008

25. Elwood JM and Jopson J: Melanoma and sun exposure: An overview of published studies. Int J Cancer 73: 198-203, 1997.

26. Ley RD and Reeve VE: Chemoprevention of ultraviolet radiationinduced skin cancer. Environ Health Perspect 105 (Suppl 4): 981-984, 1997.

27. MacKie RM: Long-term health risk to the skin of ultraviolet radiation. Prog Biophys Mol Biol 92: 92-96, 2006.

28. Harrison GI and Young AR: Ultraviolet radiation-induced erythema in human skin. Methods 28: 14-19, 2002.

29. Sliney DH: Exposure geometry and spectral environment determine photobiological effects on the human eye. Photochem Photobiol 81: 483-489, 2005.

30. Suh MH, Kwon JW, Wee WR, Han YK, Kim JH and Lee JH: Protective effect of ascorbic Acid against corneal damage by ultraviolet B irradiation: A pilot study. Cornea 27: 916-922, 2008.

31. Vink AA, Yarosh DB and Kripke ML: Chromophore for UV-induced immunosuppression: DNA. Photochem Photobiol 63: 383-386, 1996.

32. Norval M: Effects of solar radiation on the human immune system. J Photochem Photobiol B 63: 28-40, 2001.
33. Hsieh HY, Lee WC, Senadi GC, Hu WP, Liang JJ, Tsai TR, Chou YW, Kuo KK, Chen CY and Wang JJ: Discovery, synthetic methodology, and biological evaluation for antiphotoaging activity of bicyclic[1,2,3]triazoles: In vitro and in vivo studies. J Med Chem 56: 5422-5435, 2013.

34. Wlaschek M, Tantcheva-Poór I, Naderi L, Ma W, Schneider LA, Razi-Wolf Z, Schüller J and Scharffetter-Kochanek K: Solar UV irradiation and dermal photoaging. J Photochem Photobiol B 63: 41-51, 2001

35. Jung YR, Kim DH, Kim SR, An HJ, Lee EK, Tanaka T, Kim ND Yokozawa T, Park JN and Chung HY: Anti-wrinkle effect of magnesium lithospermate B from Salvia miltiorrhiza BUNGE: Inhibition of MMPs via NF-kB signaling. PLoS One 9: e102689, 2014.

36. Staniforth V, Huang WC, Aravindaram K and Yang NS: Ferulic acid, a phenolic phytochemical, inhibits UVB-induced matrix metalloproteinases in mouse skin via posttranslational mechanisms. J Nutr Biochem 23: 443-451, 2012.

37. Dërmaku-Sopjani M, Kolgeci S, Abazi S and Sopjani M: Significance of the anti-aging protein Klotho. Mol Membr Biol 30: 369-385, 2013.

38. Sopjani M, Rinnerthaler M, Kruja J and Dermaku-Sopjani M: Intracellular signaling of the aging suppressor protein Klotho. Curr Mol Med 15: 27-37, 2015

39. Lim SW, Jin L, Luo K, Jin J, Shin YJ, Hong SY and Yang CW: Klotho enhances FoxO3-mediated manganese superoxide dismutase expression by negatively regulating PI3K/AKT pathway during tacrolimus-induced oxidative stress. Cell Death Dis 8: e2972, 2017.

40. Wang Y, Kuro-o M and Sun Z: Klotho gene delivery suppresses Nox 2 expression and attenuates oxidative stress in rat aortic smooth muscle cells via the cAMP-PKA pathway. Aging Cell 11: 410-417, 2012.

41. Xie B, Cao K, Li J, Chen J, Tang J, Chen X, Xia K, Zhou X, Cheng Y, Zhou J, et al: Hmgb1 inhibits Klotho expression and malignant phenotype in melanoma cells by activating NF- $\kappa \mathrm{B}$. Oncotarget 7: 80765-80782, 2016.

This work is licensed under a Creative Commons Attribution-NonCommercial-NoDerivatives 4.0 International (CC BY-NC-ND 4.0) License. 